\title{
Robustness of the reinsurance policy: minimizing the probability of ruin
}

\author{
Bing Liu \\ School of Insurance \\ Central University of Finance and Economics
}

\begin{abstract}
In this paper, we consider the optimal reinsurance problem for an insurer who worries about the existence of the ambiguity with the objective of minimizing the probability of ruin. We assume that the surplus of the insurer is described by a diffusion process. By the dynamic programming principle, we obtain the HJB equation of the value function and give the closed form of the robust reinsurance policy and value function. We can see that the robust reinsurance policy is same as the optimal reinsurance policy in Schmidli (2001), while the value functions are different.
\end{abstract}

Keywords: Ambiguity; reinsurance policy; penalty function; probability of ruin; relative entropy

\section{INTRODUCTION}

We know that reinsurance is an important way for an insurer to avoid large losses. Therefore, the optimal reinsurance problem has been studied for decades. For example, Schmidli(2001) and Promislow and Young (2005) studied the optimal reinsurance by minimizing the ruin probability; H申jgaard and Taksar (1998) studied optimal proportional reinsurance with transaction costs by maximizing the expected present value of cash reserve process up to the ruin time. H申jgaard and Taksar (2004) investigated the optimal reinsurance problem with maximizing the expected utility of terminal wealth.

In the above works, a basic assumption behind is that the insurer should know that the accurate probability measure $P$ (we call this $P$ as reference probability measure or reference model). However, it is well know that the $P$ is constructed by the insurer's limited information. So the $P$ maybe somewhat ambiguity. Therefore, if the insurer wants to obtain the credible policies, he should consider the existence of the ambiguity. Intuitively, the insurer should not only consider the reference model but also should consider alternative probability measures. Actually, many researchers have paid attention to such problems, for example, see Hansen and Sargent (2001), Uppal and Wang (2003), Maenhout (2004), Zhang et al. (2009), Yi et al. (2013), Zeng et al. (2016).

In our work, we use a diffusion model to describe the surplus of an insurance company. We assume that the $P$ used in constructing the surplus has some ambiguities. Thus the diffusion model is not accurate. Under this circumstance, by minimizing the probability of ruin, we give the robust problem. By the principle of dynamic programming, we obtain the HJB equation of the value function and give the closed-form solutions to the robust reinsurance policy and value function.

The remainder of this paper is as follows. In Section 2, we give the model used in this paper, describe the objective function in Schmidli (2001), present some results in Schmidli (2001); In Section 3, we discuss the existence of ambiguity and give the robust control problem of this 
paper. In Section 4, we obtain the closed-form solutions of optimal policy and value function for the problem.

\section{THE MODEL}

We describe the surplus of an insurance company at time $t$ by $S(t)$

$$
\mathrm{d} S(t)=\alpha \mathrm{d} t+\beta \mathrm{d} B(t), \quad S(0)=x,
$$

where $>0, \quad>0 ; B(t)$ is a standard Brownian motion. Reinsurance is a business contract that an insurer pays premium to a reinsurer who undertakes a correspondent loss from the insurer. It is assumed that the insurer takes a proportional reinsurance to control its risk exposure. Then, with a reinsurance policy, the surplus of the insurance company can be written as

$$
\mathrm{d} S_{1}(t)=\left[\alpha a_{t}-\theta\left(1-a_{t}\right) \alpha\right] \mathrm{d} t+a_{t} \beta \mathrm{d} B(t), \quad S_{1}(0)=x,
$$

where $0<a_{t}<1$ is the proportion of total risk retained by the insurer, and is the proportional cost rate for the reinsurance. Actually, we assume that $>0$ which means that the reinsurance is non-cheap.

Next, we definite the ruin time by

$$
\tau=\inf \left\{t: S_{1}(t) \leq 0\right\}
$$

In Schmidli (2001), the value function is

$$
V(x)=\inf _{a_{t}} P\left(\tau<\infty \mid S_{1}(0)=x\right),
$$

where $P$ is the reference probability measure. The optimal reinsurance policy in Schmidli (2001) is that

$$
a_{t}^{*}=\left\{\begin{array}{cc}
\frac{2 \theta}{1+\theta}, & 0<\theta<1 \\
1, & \theta \geq 1 .
\end{array}\right.
$$

And the corresponding value function is $V(x)=\exp (k x)$, where

$$
k=\left\{\begin{array}{cc}
-\frac{(1+\theta)^{2}}{2 \beta^{2} \theta}, & 0<\theta<1 ; \\
-\frac{2 \alpha}{\beta^{2}}, & \theta \geq 1 .
\end{array}\right.
$$

A basic assumption behind problem (2.2) is that the insurer knows exactly about true probability measure $P$ used in computing above equation. However, some researchers have argued that the assumption is too strong. The insurer should be allowed to consider the optimal policies for model ambiguity. Therefore, there are many investigators have studied the optimal policies with model ambiguity. For example, see Hansen and Sargent (2001), Uppal and Wang (2003). As a result, we will consider the optimal reinsurance policy with model ambiguity. In the following subsection, we present the model ambiguity in our optimal control problems. 


\section{ROBUST CONTROL PROBLEM}

As mentioned before we should consider the alternative probability measures. Since $P$ has positive reference value, the alternative models considered by insurer should similar to the reference model, so we define the alternative models by a class of probability measures which are equivalent to $P$,

$$
\mathbb{Q}:=\{Q \mid Q \sim P\}
$$

Since $Q \mathbb{I}$ is equivalent to $P$, applying the Girsanov's theorem ( Klebaner (2008)), $Q$ satisfies

$$
\frac{\mathrm{d} Q}{\mathrm{~d} P}(B[0, T])=\Lambda(T),
$$

where

$$
\Lambda(t)=\exp \left(\int_{0}^{t} n(s) \mathrm{d} B(s)-\frac{1}{2} \int_{0}^{t}[n(s)]^{2} \mathrm{~d} s\right)
$$

is a $P$-martingale with filtration $\left\{F_{t}\right\}_{t[0, T]}, n(t)$ is a regular adapted process satisfying Novikov's condition, i.e.,

$$
E^{P}\left[\exp \left(\frac{1}{2} \int_{0}^{T}[n(s)]^{2} \mathrm{~d} s\right)\right]<\infty .
$$

By Girsanov's theorem, the standard Brownian motion $B(t)$ under probability measure $P$ can be represented as

$$
\mathrm{d} B(t)=n(t) \mathrm{d} t+\mathrm{d} B^{Q}(t),
$$

where $B^{Q}(t)$ is a standard Brownian motion under probability measure $Q$.

We should derive the wealth process under $Q$. Inserting (3.2) and into (2.1), we have

$$
\mathrm{d} S_{1}^{Q}(t)=\left[a_{t} \alpha-\theta\left(1-a_{t}\right) \alpha+a_{t} \beta n(t)\right] \mathrm{d} t+a_{t} \beta \mathrm{d} B^{Q}(t), \quad S_{1}^{Q}(0)=x .
$$

In order to consider the alternative probability measure $Q$, we should measure the discrepancy between each alternative probability measure and reference probability measure by relative entropy. The relative entropy is a well-established approach in measuring the discrepancy between $Q$ and $P$, for example, see Uppal and Wang (2003), Yi et al. (2013). The relative entropy between $\mathrm{n} Q$ and $P$ is given by 


$$
\begin{aligned}
H_{[0, t]}(Q \| P)=E_{[0, t]}^{Q}\left[\ln \frac{\mathrm{d} Q}{\mathrm{~d} P}\right] & =E^{Q}\left[\int_{0}^{t} n(s) \mathrm{d} B(s)-\frac{1}{2} \int_{0}^{t}[n(s)]^{2} \mathrm{~d} s\right] \\
& =E^{Q}\left[\int_{0}^{t} n(s) \mathrm{d} B^{Q}(s)+\frac{1}{2} \int_{0}^{t}[n(s)]^{2} \mathrm{~d} s\right]
\end{aligned}
$$

Since $B^{Q}(t)$ is a standard Brownian motion under probability measure $Q$, we have

$$
H_{[0, t]}(Q \| P)=E^{Q}\left[\int_{0}^{t} Z(s) \mathrm{d} s\right]=E^{Q}\left[\int_{0}^{t} \frac{1}{2}[\mathrm{n}(\mathrm{s})]^{2} \mathrm{~d} s\right]
$$

where $Z(s)=\frac{1}{\imath}[n(s)]^{2}$. Hence, $Z(t)$ measures the $H_{[0, t]}(Q \| P)$.

Since $P$ has positive reference value. Thus, if the insurer doesn't use the reference probability measure $P$ and uses the alternative probability measure $Q$, a penalty will occur. intuitively, the larger the $H_{[0, t]}(Q \| P)$ is, the larger the penalty will occur. Consequently, we formulate a robust control problem in the following. We first define ruin time

$$
\tau^{Q}=\inf \left\{t: S_{1}^{Q}(t) \leq 0\right\} \text {. }
$$

For a policy $0<a<1$, the objective of minimizing the probability of ruin with ambiguity aversion is set as

$$
V^{Q}(x)=\inf _{a} \sup _{Q \in \mathbb{Q}}\left\{Q_{x}\left(\tau^{Q}<\infty\right)-E_{x}^{Q}\left[\int_{0}^{\tau^{Q}} \xi \phi\left(V^{Q}(x)\right) Z(s) \mathrm{d} s\right]\right\},
$$

where $Q_{x}()=Q\left(\mid S_{1}^{Q}(0)=x\right) ; E_{x}^{Q}[]=E^{Q}\left[\mid S_{1}^{Q}(0)=x\right] ; x \quad 0$, ( )>0 is a standardization function that converts the penalty to the same order of magnitude as the order of $V^{Q}(x) ; Z(t)$ measures relative entropy, $>0$ denotes the insurer's confidence on reference model $P$, the larger the is, the insurer has more confidence on $P$; the sup term reflects the insurer's aversion to ambiguity, in other words, the insurer is conservative and then he will consider the worst result with ambiguity. In the extreme case, means that the insurer is extremely confident about the reference model and the use of any alternative model will incur the heavily penalty. On the other hand, 0 means that the insurer has no information about the reference model. Hence, we assume $0<<1$ in the following study.

\section{ROBUST POLICY}

We can easily to get that the value function $V^{Q}(x)$ of (3.4) satisfies that $V^{Q}(x)>0$ $\frac{\partial}{\partial x} V^{Q}(x)>0, \frac{\partial^{2}}{\partial x^{2}} V^{Q}(x)<0$. According to the principle of dynamic programming, we can obtain the Hamilton-Jacobi-Bellman (HJB) equation of value function (see Fleming and Soner (2006))

$$
\inf _{a} \sup _{n}\left\{\left[\alpha a_{t}-\theta\left(1-a_{t}\right) \alpha+a_{t} \beta n\right] \frac{\partial}{\partial x} V^{Q}(x)+\frac{1}{2} a_{t}^{2} \beta^{2} \frac{\partial^{2}}{\partial x^{2}} V^{Q}(x)-\frac{1}{2} \xi \phi\left(V^{Q}(x)\right) n^{2}\right\}=0,
$$


with the boundary condition $V^{Q}(0)=1$.

Theorem 4.1 (Verification Theorem) If $(x) \quad \mathbb{C}^{2}$ ( twice differentiable) is the solution to HJB equation (4.1) with the boundary condition $(0)=1$, then $(x)=V^{Q}(x)$.

Proof. The proof is standardized, we omit it here.

Furthermore, as mentioned before, in order to converts the penalty to the same order of magnitude as the order of $V^{Q}(x)$, we choose a suitable form of ()

$$
\phi\left(V^{Q}(x)\right)=V^{Q}(x) \text {. }
$$

Substituting (4.2) into (4.1), we have

$$
\inf _{a} \sup _{n}\left\{\left[\alpha a_{t}-\theta\left(1-a_{t}\right) \alpha+a_{t} \beta n\right] \frac{\partial}{\partial x} V^{Q}(x)+\frac{1}{2} a_{t}^{2} \beta^{2} \frac{\partial^{2}}{\partial x^{2}} V^{Q}(x)-\frac{1}{2} \xi V^{Q}(x) n^{2}\right\}=0 \text {. }
$$

Since $-\frac{1}{2} \xi V^{Q}(x)<0$, in accordance with the first-order conditions, $n^{*}$ satisfies the sup term of (4.3) that has the following form

$$
n^{*}=\frac{a_{t} \beta \frac{\partial}{\partial x} V^{Q}(x)}{\xi V^{Q}(x)} .
$$

Putting (4.4) into (4.3), yields

$$
\inf _{a}\left\{\left[\alpha a_{t}-\theta\left(1-a_{t}\right) \alpha+a_{t} \beta n\right] \frac{\partial}{\partial x} V^{Q}(x)+\frac{1}{2} a_{t}^{2} \beta^{2} \frac{\partial^{2}}{\partial x^{2}} V^{Q}(x)-\frac{1}{2} \frac{a_{t}^{2} \beta^{2}\left[\frac{\partial}{\partial x} V^{Q}(x)\right]^{2}}{\xi V^{Q}(x)}\right\}=0 .
$$

With the boundary condition $V^{Q}(0)=1$, we speculate that the value function has the following form

$$
V^{Q}(x)=\exp (K x)
$$

where $K$ is a parameter will be given explicitly later.

Then, we have

$$
\begin{gathered}
\frac{\partial}{\partial x} V^{Q}(x)=K V^{Q}(x), \\
\frac{\partial^{2}}{\partial x^{2}} V^{Q}(x)=K^{2} V^{Q}(x) .
\end{gathered}
$$

Substituting (4.6) and (4.7) into (4.5), we have

$$
\inf _{a}\left\{\left[\alpha a_{t}-\theta\left(1-a_{t}\right) \alpha+a_{t} \beta n\right]+\frac{1}{2} a_{t}^{2} \beta^{2} K+\frac{1}{2} \frac{a_{t}^{2} \beta^{2} K}{\xi}\right\}=0 \text {. }
$$

According to first-order conditions, we have 


$$
a_{t}^{\text {star }}=\frac{-(1+\theta) \alpha}{\beta^{2}\left(1+\frac{1}{\xi}\right) K}
$$

Inserting (4.9) into (4.8), we obtain

$$
-\frac{1}{2} \frac{(1+\theta)^{2} \alpha^{2}}{\beta^{2}\left(1+\frac{1}{\xi}\right) K}-\alpha \theta=0 .
$$

Thus, we have

$$
K=-\frac{1}{2} \frac{(1+\theta)^{2} \alpha}{\beta^{2}\left(1+\frac{1}{\xi}\right) \theta} .
$$

Substituting (4.10) into (4.9), yields

$$
a_{t}^{\mathrm{star}}=\frac{2 \theta}{1+\theta}
$$

Case (i) For $0<<1$

In this case, we can see that $0<a_{t}^{\text {star }}<1$. Therefore, the robust reinsurance policy is $a^{*}=a^{\text {star }}$

\section{Case (ii) For 1}

In this case, we can see that $a^{\text {star }} \quad 1$. Therefore, the robust reinsurance policy is $a^{*}=1$.

Substituting $a^{*}=1$ into (4.8), we have the corresponding $K=\frac{2}{{ }^{2}\left(1+\frac{1}{-}\right)}$. Therefore, the robust

reinsurance policy can be written as

$$
a_{t}^{*}=\left\{\begin{array}{cc}
\frac{2 \theta}{1+\theta}, & 0<\theta<1 ; \\
1, & \theta \geq 1 .
\end{array}\right.
$$

Before we give the follow theorem, we also need to verify that the Novikov's condition is satisfied. In other words, we should guarantee $(T)$ with $n^{*}$ is a P-martingale. Hence, we should have

$$
E\left[\exp \left(\frac{1}{2} \int_{0}^{T}[n(t)]^{2} \mathrm{~d} s\right)\right]<\infty .
$$

That is

$$
E\left[\exp \left(\frac{1}{2} \int_{0}^{T}\left[\frac{a_{t} \beta \frac{\partial}{\partial x} V^{Q}(x)}{\xi V^{Q}(x)}\right]^{2} \mathrm{~d} s\right)\right]<\infty
$$

which equivalent to

$$
E\left[\exp \left(\frac{1}{2} \int_{0}^{T}\left[\frac{a_{t}^{*} \beta K}{\xi}\right]^{2} \mathrm{~d} s\right)\right]<\infty
$$


we know that $a_{t}^{*}<, K<$ and other parameters are constants, therefore (4.12) is satisfied. The above results can be summarized as the following theorem.

Theorem 4.2 For the problem (3.4) with (4.2), we can obtain the robust reinsurance policy as follows.

$$
a_{t}^{*}=\left\{\begin{array}{cc}
\frac{2 \theta}{1+\theta}, & 0<\theta<1 ; \\
1, & \theta \geq 1 .
\end{array}\right.
$$

The corresponding value function can be written as $V^{Q}(x)=\exp (K x)$, where

$$
K=\left\{\begin{array}{cc}
-\frac{1}{2} \frac{(1+\theta)^{2} \alpha}{\beta^{2}\left(1+\frac{1}{\xi}\right) \theta}, & 0<\theta<1 ; \\
-\frac{2 \alpha}{\beta^{2}\left(1+\frac{1}{\xi}\right)}, & \theta \geq 1 .
\end{array}\right.
$$

Remark It is interesting that the robust reinsurance policy here is same as the Schmidli's (2001) optimal reinsurance policy. In Schmidli's (2001), they do not consider the existence of ambiguity.

\section{References}

Fleming, W.H., Soner, M. (2006). Controlled Markov Processes and Viscosity Solutions, 2nd edition. Springer, New York.

Hansen, L. P., Sargent, T. J. (2001). Robust control and model uncertainty. American Economic Review 91(2), 6066.

Højgaard, B., Taksar, M., (1998). Optimal proportional reinsurance policies for diffusion models with transaction costs. Insurance Math. Econom. 22,41-51.

Højgaard, B., Taksar, M., (2004). Optimal dynamic portfolio selection for a corporation with controllable risk and dividend distribution policy. Quant. Finance, 4, 315-327.

Klebaner, F. C. (2008). Introduction to stochastic calculus with applications. IMPERIAL COLLEGE PRESS.

Maenhout, P. J. (2004). Robust portfolio rules and asset pricing. Review of Financial Studies 17(4), 951-983.

Promislow, D.S., Young, V.R., (2005). Minimizing the probability of ruin when claims follow Brownian motion with drift. N. Am. Actuar. J. 9(3),109-128.

Schmidli, H. (2001). Optimal proportional reinsurance policies in a dynamic setting. Scandinavian Actuarial Journal 1, 55-68.

Schmidli, H. (2002). On minimizing the ruin probability by investment and reinsurance. The Annals of Applied Probability 12, 890-907.

Uppal, R., Wang, T. (2003). Model misspecification and underdiversification. The Journal of Finance 58(6), 24652486.

Yi, B., Li, Z., Viens, F. G., Zeng, Y. (2013). Robust optimal control for an insurer with reinsurance and investment under hestons stochastic volatility model. Insurance Mathematics and Economics 53(3), 601-614.

Zeng, Y., Li, D., Gu, A. (2016). Robust equilibrium reinsurance-investment strategy for a mean-variance insurer in a model with jumps. Insurance Mathematics and Economics 66, 138-152.

Zhang, X., Zhang, K., Yu, X. (2009). Optimal proportional reinsurance and investment with transaction costs, I: Maximizing the terminal wealth. Insurance: Mathematics and Economics 44, 473-478. 